\title{
Wingspan stenting of symptomatic extracranial vertebral artery stenosis and perioperative evaluation using quantitative magnetic resonance angiography: report of two cases
}

\author{
Jonathan L. BRisman, M.D. \\ Department of Cerebrovascular and Endovascular Neurosurgery, Winthrop University Hospital, \\ Mineola, Long Island, New York
}

\begin{abstract}
$\checkmark$ The best management strategy for symptomatic vertebrobasilar ischemia is currently not well-defined. Noninvasive Optimal Vessel Analysis (NOVA, VasSol, Inc.) is computer software that, using quantitative magnetic resonance (MR) angiography technology, represents the only commercially available means of noninvasively measuring blood flow within the human vasculature.

The author used quantitative MR angiography to study cerebral blood flow in 2 patients who underwent angioplasty and stenting for medically refractory extracranial cervical vertebral artery (VA) stenosis using the recently Food and Drug Administration-approved WingSpan stent (Boston Scientific, Target). WingSpan stents were successfully placed after balloon angioplasty in both patients without complications. At the 5-month clinical follow-up examination, 1 patient was symptom free and the other had had a possible transient ischemic attack without sequelae. The WingSpan stent may represent an alternative management scheme for symptomatic vertebrobasilar ischemia from extracranial VA stenosis. Quantitative MR angiography can readily measure blood flow in the vertebrobasilar system, and these values correlated with the angiographic outcomes in the 2 patients treated in the present study.
\end{abstract}

(DOI: $10.3171 /$ FOC/2008/24/2/E14)

\author{
KeY WORDS • angioplasty - NOVA - quantitative MR angiography • \\ vertebral artery - WingSpan stent
}

$\mathrm{V}$ ERTEBROBASILAR ischemia from stenoocclusive VA or BA disease represents a serious disorder with a high rate of death and disability if left untreated. ${ }^{9}$ Treatment has historically included the use of antiplatelet or antithrombotic agents, often associated with high rates of recurrent ischemia and significant complications. Surgical, and more recently, endovascular approaches have therefore been used in an effort to treat this disorder with its well-defined natural history, particularly in patients whose condition worsens despite maximal medical therapy.

Stenoses or occlusions anywhere from the vertebral ostia to the basilar apex may contribute to vertebrobasilar ischemia, the manifestations of which are intimately related to each patient's unique collateral blood supply and cerebrovascular reserve. An individual's capacity to compensate for vertebrobasilar narrowing and/or atherosclerotic disease is difficult to quantify because current cerebrovas-

Abbreviations used in this paper: $\mathrm{BA}=$ basilar artery; $\mathrm{CT}=$ computed tomography; $\mathrm{MR}=$ magnetic resonance; NOVA = Noninvasive Optimal Vessel Analysis; VA = vertebral artery. cular reserve tests appear to be significantly more sensitive and specific when applied to the anterior circulation. ${ }^{1}$ The inability to predict which patients with vertebrobasilar ischemia are at the highest risk for cerebral ischemia-which can often be permanently disabling given the vascular territory supplied-have made management of this disorder frustrating. Nonmedical options have moved from surgical bypass $^{10}$ to the use of coronary balloon-mounted stents or other stents designed for nonintracranial use, with not insignificant rates of procedural complications and high rates of in-stent restenosis. ${ }^{5,8,11}$ The WingSpan stent (Boston Scientific, Target) was recently approved by the Food and Drug Administration (approval granted as a human-use device in 2005) for medically refractory intracranial stenoses. The self-expanding nitinol stent (Fig. 1) is designed for use after balloon angioplasty using a specially designed balloon (Gateway balloon, Boston Scientific, Target) and has not been previously described in the treatment of extracranial VA disease.

Quantitative MR angiography is currently the only noninvasive modality with which to quantify blood flow in the human vasculature. Few reports on the clinical use of this 


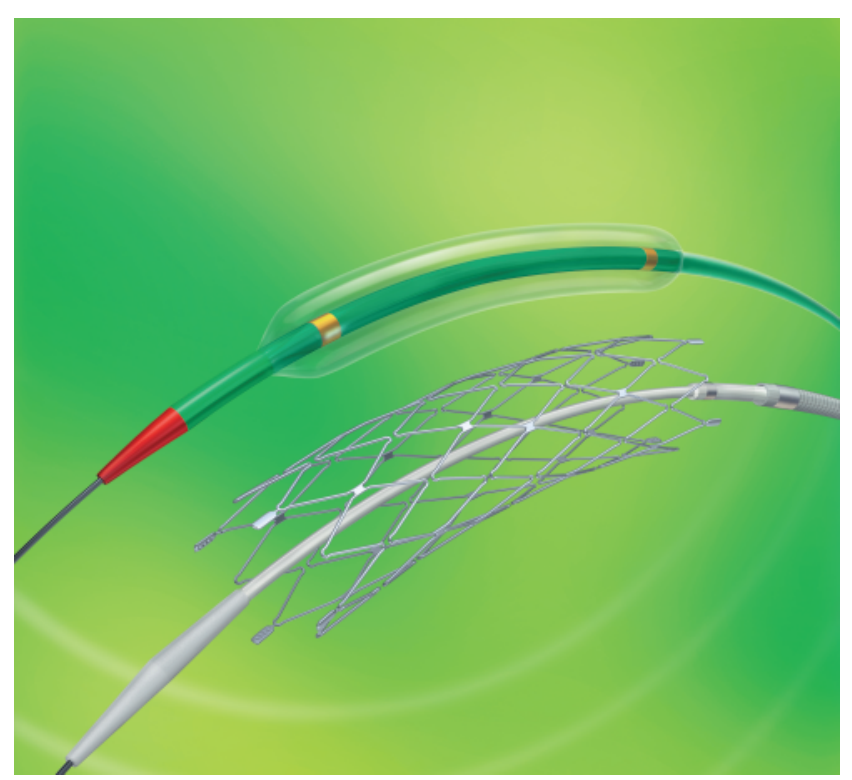

FIG. 1. Illustration of the Gateway balloon (upper) and Wingspan stent system.

technology exist. VasSol, Inc. acquired premarket Food and Drug Administration approval for their NOVA software in 2002. Reports on NOVA have been published demonstrating its use as a decision-making tool in patients with vertebrobasilar ischemia, ${ }^{1}$ as a means to document vascular bypass patency, ${ }^{2}$ as a measure of successful embolization of vein of Galen malformations, ${ }^{7}$ as a means to determine whether patients will tolerate carotid occlusion, ${ }^{4}$ and as a means to understand a difficult case of hemispheric ischemia and subclavian stenosis and plan the treatment. ${ }^{6}$ Its use in association with the WingSpan stent has not been previously described.

\section{Case Reports}

\section{Case 1}

This 52-year-old man with a history of smoking cigarettes, hypertension, hypercholesterolemia, and coronary artery disease had recurrent episodes of diplopia associated with left hemisensory symptoms. These symptoms had not been previously associated with strokes, and he had been prescribed a regimen of clopidogrel and aspirin. On his most recent admission, the patient again complained of double vision and left arm and leg numbness. The results of his examination were unremarkable except for some endgaze nystagmus on leftward gaze and subjective decreased sensation in the left arm and leg. Magnetic resonance imaging demonstrated acute infarcts of the bilateral occipital lobes, left cerebellum, and right pons (Fig. 2A). Computed tomography angiography of the head, aortic arch, and neck showed multiple extracranial stenoses of both VAs. Quantitative MR angiography demonstrated markedly diminished blood flow in the BA and both VAs (Fig. 2B, C).

After general anesthesia had been induced, the patient underwent diagnostic angiography which confirmed multiple extracranial VA stenoses, including severe narrowing of the right VA origin (Fig. 2D) and multiple areas along the left VA in the high cervical region (Fig. 2E). In the most stenotic area there was severe stenosis with irregularity suggestive of an ulcerated plaque. Very sluggish flow into the basilar system was seen. Under full heparinization, a 6F Envoy catheter was navigated into the left VA, and a Gateway Balloon measuring $3.5 \times 15 \mathrm{~mm}$ was advanced over a 0.14-inch microguidewire across the lesion and inflated. After angioplasty was performed, 2 WingSpan stents $(4 \times 15$ and $3.5 \times 15 \mathrm{~mm})$ were loaded onto the same wire and deployed in a telescoping fashion across the area of stenosis with good angiographic reconstruction of the affected segment (Fig. 2F, G). Markedly increased flow into the BA was seen. The poststenting quantitative MR angiography study documented significantly increased flow into the left VA and BA (Fig. 2H). The patient was continued on a regimen of clopidogrel and discharged to a rehabilitation facility without further episodes. He was readmitted 4 months later with transient facial numbness, but CT angiography failed to reveal in-stent stenosis and MR imaging did not show an acute infarction.

\section{Case 2}

This 69-year-old man presented with a history of hypertension, hyperlipidemia, and diabetes. Three months prior to the current admission he had had an episode of vertigo and was found to have a right cerebellar and a very small left cerebellar acute infarction (Fig. 3A). He made a good recovery and was discharged on a regimen of clopidogrel. He was readmitted with episodes of diplopia and a feeling of unbalance while walking. Magnetic resonance imaging showed small bilateral cerebellar and occipital lobe acute infarctions. Computed tomography angiography revealed a very focal area of stenosis of the VA a small distance from the entrance into the foramen magnum. Quantitative MR angiography showed markedly diminished flow in the right VA compared with the left and normal basilar flow (Fig. 3B, C). The patient underwent diagnostic angiography, which confirmed a single very severe stenosis just proximal to the entrance of the VA into the posterior fossa (Fig. 3D, E).

Under full heparinization, a 6-F Envoy was navigated into the right VA, and the Gateway balloon measuring $3.5 \times 15 \mathrm{~mm}$ was used to perform angioplasty of the stenotic segment. Using the same 0.14-inch microguidewire for support, a WingSpan stent measuring $3 \times 15 \mathrm{~mm}$ was deployed across the stenosis with good results on angiography (Fig. 3F). Quantitative MR angiography documented increased flow in the right VA (Fig. 3G, H). The patient has remained symptom-free at the 5-month followup examination.

\section{Discussion}

This report documents the utilization of the new WingSpan stent and Gateway balloon system to treat 2 patients with medically refractory vertebrobasilar ischemia secondary to cervical VA stenosis. This is the first description of the use of this novel stent to treat extracranial VA stenosis as well as the first documentation of the use of quantitative MR angiography before and after WingSpan stenting of any vessel.

Historically, extracranial VA disease had been treated 

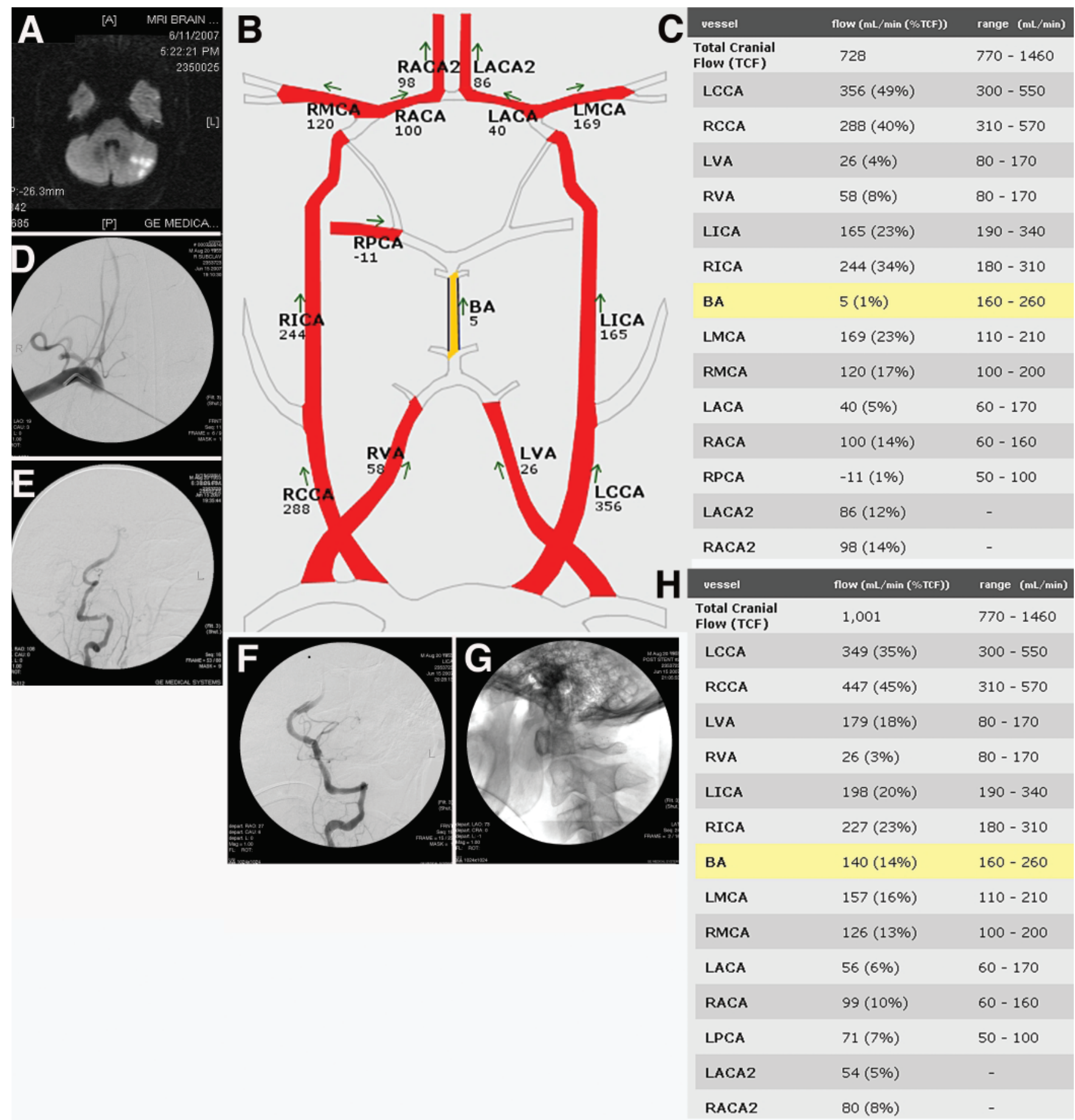

FIG. 2. Case 1. A: Diffusion-weighted brain MR image demonstrating acute left cerebellar hemisphere infarction. B: Quantitative MR angiography (NOVA) flow map showing markedly diminished flow in both VAs (26 and 58 $\mathrm{ml} / \mathrm{minute})$ and in the $\mathrm{BA}(5 \mathrm{ml} / \mathrm{min})$. ACA = anterior cerebral artery; CCA = common carotid artery; ICA = internal carotid artery; L = left; MCA = middle cerebral artery; R = right. Arrows indicate directionality of blood flow. C: Quantitative MR angiography (NOVA) flow table giving the values seen in the flow map with the "range" column representing expected normal values. D: Angiogram of the right subclavian artery, posteroanterior view, demonstrating severe right VA origin stenosis. E: Magnified oblique view of the left VA angiogram demonstrating stenotic irregular plaque in the high cervical VA. F: Oblique view of the left VA angiogram demonstrating successful angiographic reconstruction poststent placement. G: Lateral X-ray film of the cervical spine demonstrating the 2 telescoping WingSpan stents placed at the C-1 level of the VA. H: Quantitative MR angiography (NOVA) flow table demonstrating the marked increase in the left VA flow after stenting with resultant increase in basilar flow. 

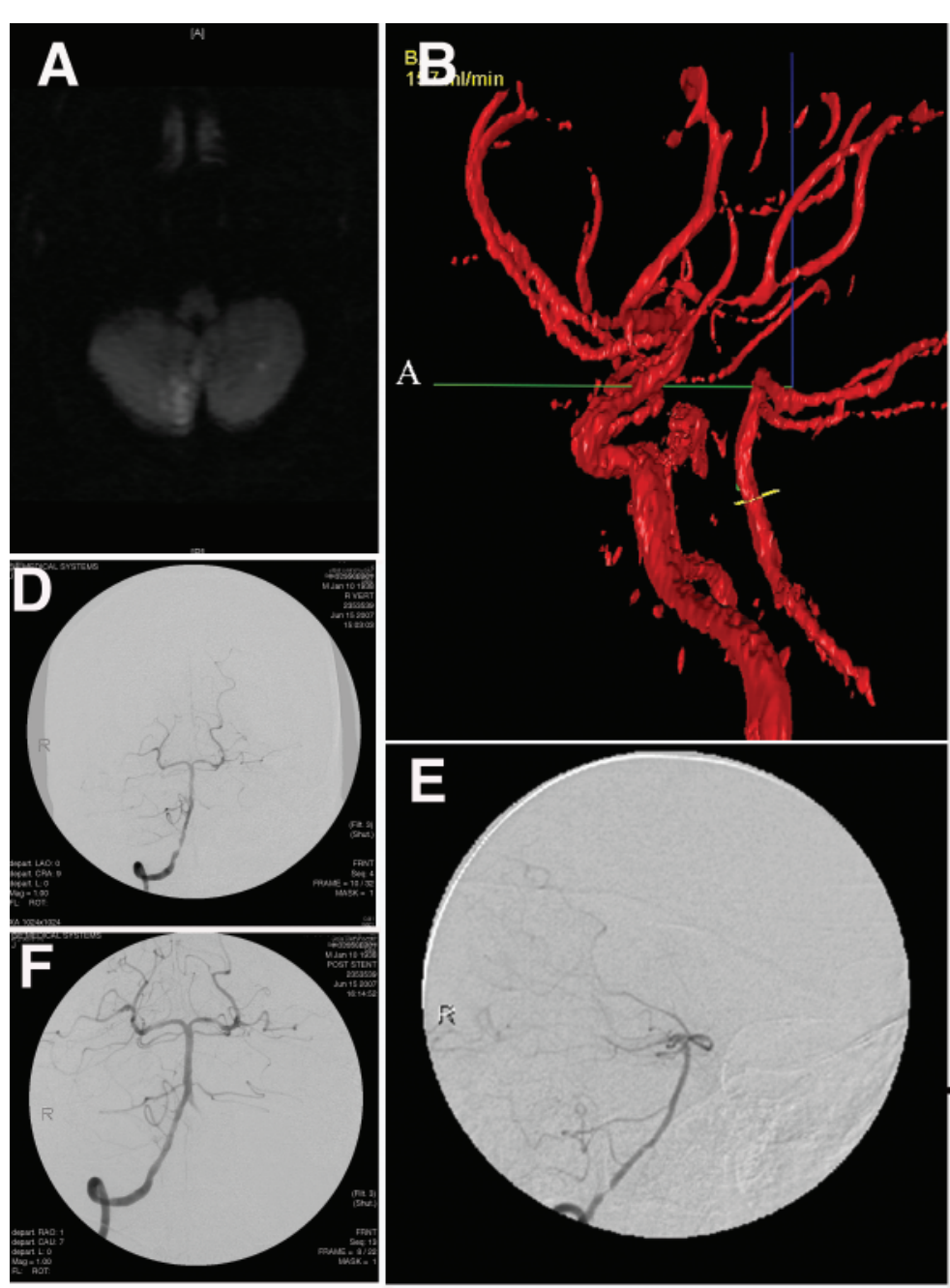

\begin{tabular}{|lll|}
\hline LVA & 189 & $80-170$ \\
\hline RVA & 73 & $80-170$ \\
\hline LICA & 366 & $190-340$ \\
\hline RICA & 214 & $180-310$ \\
\hline BA & 166 & $160-260$ \\
\hline LMCA & 177 & $110-210$ \\
\hline RMCA & 129 & $100-200$ \\
\hline
\end{tabular}

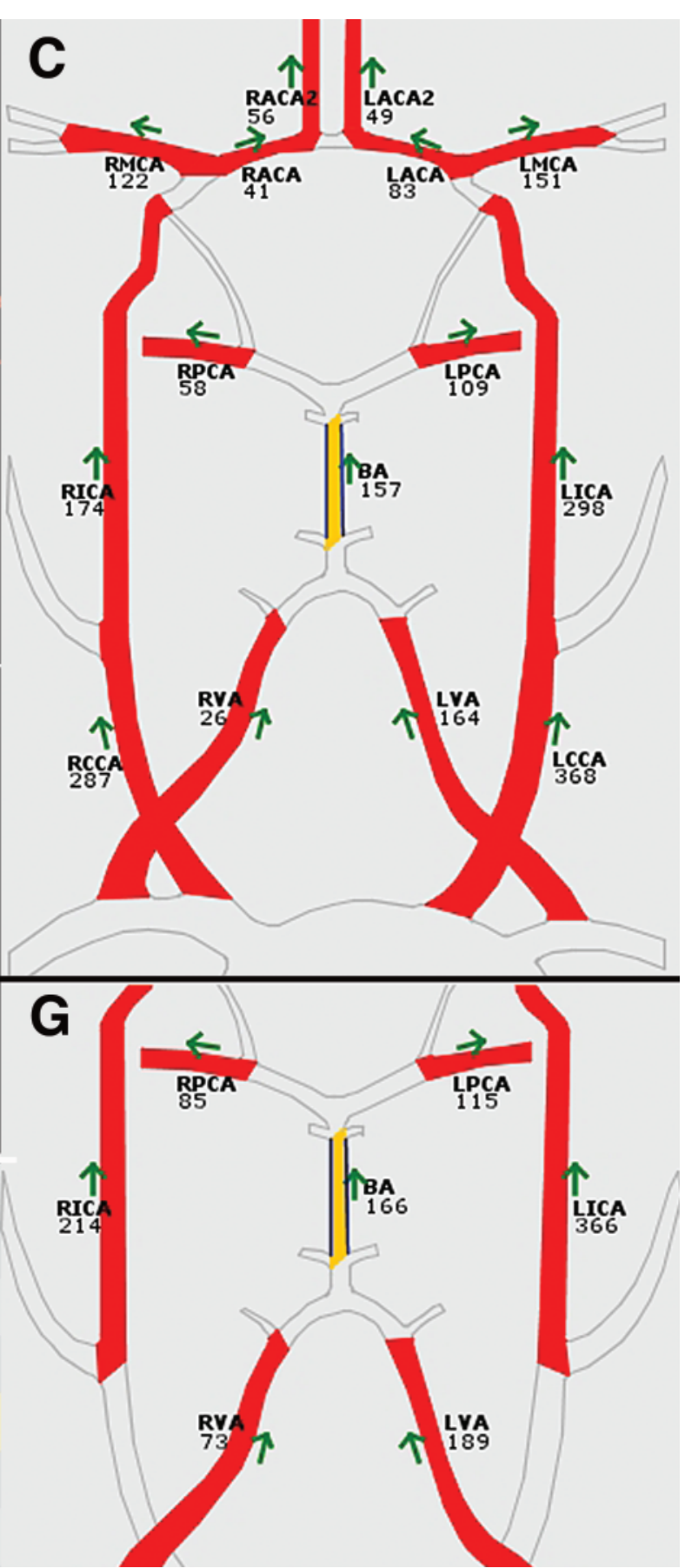

FIG. 3. Case 2. A: Diffusion weighted brain MR image showing acute right cerebellar medial hemispheric infarction. B: Noninvasive Optimal Vessel Analysis MR angiogram shows the region in the BA (yellow line) where the flow was measured. Flow is normal (157 $\mathrm{ml} /$ minute). C: Quantitative MR angiogram (NOVA) flow map shows very low flow in the right VA (26 ml/minute) compared with the nonstenotic left VA (164 ml/minute). Again, normal flow in the BA is seen. D: Right VA posteroanterior angiogram demonstrates severe right VA stenosis proximal to its entrance intracranially. E: Right VA angiogram, lateral view. F: Right VA angiogram, magnified lateral view, demonstrating good angiographic results postangioplasty and stent placement. G: Quantitative MR angiogram (NOVA) flow map shows increased flow in the right VA poststenting. H: Quantitative MR angiogram (NOVA) flow table demonstrates that the flow in the right VA poststenting approaches the expected normal values.

with surgical bypass ${ }^{10}$ but surgery has since given way to endovascular approaches, in part because of the technical difficulty and unfamiliarity of the former and the improved success and experience with the latter. Stenting and angio- plasty have been well-described for the extracranial VAs, 5,8 with some success with both balloon-mounted coronary stents and self-expanding stents designed for noncerebral revascularization. Such stents can be difficult to place due 
to the tortuosity of the cervicocerebral vasculature, and their use is associated with complication rates (a $4.8 \%$ rate of major complications in 1 series) ${ }^{8}$ that may relate to the difficulty of stent delivery, overly aggressive balloon angioplasty, and/or excessive radial force during deployment or afterwards. Recurrent events poststenting (persistent in $9.5 \%$ in 1 series $)^{8}$ and high rates of restenosis $(10-43 \%)^{11}$ suggest that current endovascular options need improvement. The WingSpan stent system and Gateway balloon were specifically designed to access and treat more fragile intracranial vessels. In the recently published seminal safety study for this stent, placement was performed in 45 patients with medically refractory intracranial stenosis. ${ }^{3}$ Although not specifically designed for extracranial disease, the system was employed with technical ease and success in the 2 patients here described.

In addition to this technical success associated with very acceptable angiographic results, quantitative MR angiography demonstrated expected increases in flow in the treated VA in both patients. That said, 1 patient did have an episode several months later of transient facial numbness that may have represented a posterior circulation transient ischemic attack. This patient, however, has multiple risk factors for vasculopathic disease and stenting was directed to the most stenotic and irregular region of the VA. If such episodes persist, consideration may be given to treatment of his additional VA stenoses. Another caveat for the use of this system is the issue of in-stent restenosis, which has been welldescribed for VA stents and was not addressed in this report. As the clinical follow-up period is short and no catheter angiographic follow-up has been presented, the durability of the WingSpan stent in the extracranial VAs remains unknown. Quantitative MR angiography may prove useful in the follow-up of patients after stent placement because decreased flow may herald restenosis.

Currently there are several very good imaging modalities with which to study the anatomy of the cervicocerebral vasculature. These include ultrasonography (carotid duplex and transcranial duplex), CT angiography, MR angiography, and catheter angiography, and they range from noninvasive (duplex, MR angiography) to minimally invasive (CT angiography) to invasive with low risk (catheter angiography). Those studies that are minimally or noninvasive, however, yield static images, and despite tremendous advances in the ability to manipulate these images, the studies do not give more than a picture of the vascular anatomy at a single point in time. Catheter angiography offers specific additional information about cervicocerebral vascular lesions such as the pace of blood flow and collateralization, but because of the invasiveness of the procedure there has been a continued search for less invasive modalities that might offer similar or better information. Additional modalities have been developed, therefore, using a wide range of techniques that attempt to assess cerebrovascular physiology, including single photon emission computed tomography, xenon CT, transcranial Doppler ultrasonography, positron emission tomography, CT perfusion, and MR perfusion scanning. None of these modalities gives quantitative information concerning blood flow. Noninvasive Optimal Vessel Analysis is a software package that represents the only commercially available technique to quantify cervicocerebral blood flow utilizing stan- dard MR imaging platforms. It has been shown to effectively risk-stratify patients with vertebrobasilar ischemia based on distal flow in the BA and posterior cerebral artery. ${ }^{1}$

In the 2 cases in the present study, postprocedural vessel dilatation was associated with an expected increase in flow values. Interestingly, in 1 patient, basilar flow was normal, suggesting that the lesion was not flow limiting. Stent placement in that patient might have more efficacy as an intimal stabilizer against further thromboembolic events, whereas in the other patient flow limitation and thromboembolism were both probably contributing to ischemia.

There are some limitations of the NOVA technology. The most prominent problem is the lack of properly defined baseline normal values. The baseline values provided by the manufacturer are based on a cohort of volunteers, and an analysis of this population has not yet been published. Particular clarification is needed with regard to defining the range of normal values and what, if any, adjustments should be made for certain patient-specific factors such as age, cardiac dysfunction, or dehydration, to name just a few. Nonetheless, this technology provides a method quantifying blood flow that may have particular importance for cases of vertebrobasilar stenosis for which current cerebrovascular reserve studies are insufficient.

\section{Conclusions}

Extracranial cervical VA symptomatic stenosis can be treated with the new WingSpan stent and Gateway balloon system with excellent technical success. Quantitative MR angiography can effectively measure flow in the vertebrobasilar system before and after such revascularization, and such measurements correlate with angiographic findings. The significance of this technology and the durability of the WingSpan stent for this indication are not yet known.

\section{Financial Disclosure}

The author reports no financial or other conflict of interest in regard to the devices/products described.

\section{References}

1. Amin-Hanjani S, Du X, Zhao M, Walsh K, Malisch T, Charbel FT: Use of quantitative magnetic resonance angiography to stratify stroke risk in symptomatic vertebrobasilar ischemia. Stroke 36:1140-1145, 2005

2. Amin-Hanjani S, Shin JH, Zhao M, Du X, Charbel FT: Evaluation of extracranial-intracranial bypass using quantitative magnetic resonance angiography. J Neurosurg 106:291-298, 2007

3. Bose A, Hartmann M, Henkes H, Liu HM, Teng MH, Szikora I, et al: A novel, self-expanding, nitinol stent in medically refractory intracranial atherosclerotic stenoses: the WingSpan Study. Stroke 38:1531-1537, 2007

4. Charbel FT, Zhao M, Amin-Hanjani S, Hoffman W, Du X, Clark ME: A patient-specific computer model to predict outcomes of the balloon occlusion test. J Neurosurg 101:977-988, 2004

5. Ko YG, Park S, Kim JY, Min PK, Choi EY, Jung JH, et al: Percutaneous interventional treatment of extracranial vertebral artery stenosis with coronary stents. Yonsei Med J 45:629-634, 2004

6. Langer DJ, Lefton DR, Ostergren L, Brockington CD, Song J, Niimi Y, et al: Hemispheric revascularization in the setting of carotid occlusion and subclavian steal: a diagnostic and manage- 
ment role for quantitative magnetic resonance angiography? Neurosurgery 58:528-533, 2006

7. Langer DJ, Song JK, Niimi Y, Chwajol M, Lefton DR, Brisman $\mathrm{JL}$, et al: Transarterial embolization of vein of Galen malformations: the use of magnetic resonance imaging noninvasive optimal vessel analysis to quantify shunt reduction. Report of two cases. $\mathbf{J}$ Neurosurg 104 (1 Suppl):41-45, 2006

8. Malek AM, Higashida RT, Phatouros CC, Lempert TE, Meyers PM, Gress DR, et al: Treatment of posterior circulation ischemia with extracranial percutaneous balloon angioplasty and stent placement. Stroke 10:2073-2085, 1999

9. Qureshi AI, Ziai WC, Yahia AM, Mohammad Y, Sen S, Agarwal $\mathrm{P}$, et al: Stroke-free survival and its determinants in patients with symptomatic vertebrobasilar stenosis: a multicenter study. Neurosurgery 52:1033-1040, 2003
10. Spetzler RF, Hadley MN, Martin NA, Hopkins LN, Carter LP, Budny J: Vertebrobasilar insufficiency. Part 1: Microsurgical treatment of extracranial vertebrobasilar disease. J Neurosurg 66:648-661, 1987

11. Wehman JC, Hanel RA, Guidot CA, Guterman LR, Hopkins LN: Atherosclerotic occlusive extracranial vertebral artery disease: indications for intervention, endovascular techniques, short-term and long-term results. J Interv Cardiol 17:219-232, 2004

Manuscript submitted November 15, 2007.

Accepted December 4, 2007.

Address correspondence to: Jonathan Brisman, M.D., Director, Cerebrovascular and Endovascular Neurosurgery, Winthrop University Hospital, 100 Merrick Road, Suite 128W, Rockville Centre, New York, 11570. email: jbrisman@ @eurosurgeryli.com. 